Had the patient consented to an operation at the time of the first attack, probably there would have been little difficulty in dealing with the appendix; but with the recurrence in 1903 a condition arose which surgery was powerless to relieve.

\section{PERFORATED DUODENAL ULCER :}

WITH A REPORT OF THREE CASES.

Bx H. S. CLOGG, M.S.Lond., F.R.C.S.EnG., Assistant Surgeon, Charing Cross Hospital ; Surgeon to In-patients,
Evelina Hospital for Sick Children.

THE three following cases of perforated duodenal ulcer which have recently been under my care present some points of interest, and hence $I$ venture to record them:

CASE I.

Male, aged 44. He had always enjoyed very good health; it was impossible to obtain any history of previous gastric or intestinal disease. Whilst lifting a heavy packing case he was suddenly seized with aoute pain in the right hypochondriac region; he became ver faint and was carried to the hospital, a distance of about roo yards. On admission he had recovered to a great extent from his initial collapse ; the pulse was of good quality, 80 per minute ; temperature $97^{\circ}$ He vomited three times in rapid succession; the vomit was bile-stained. He complained of considerable pain in the right hypochondriac region, and this region of the abdominal wall was held very rigid ; the maximum point of tenderness was about $x$ in. above and to the right of the umbilicus. Apart from the right hypochondriac region the abdomen was free from all tenderness and moved freely on respiration. No food had been taken for some two hours previous to the occurrence of the severe pain. Two or three hours later the pain was much easier; he again vomited some bile-stained material. The temperature had risen to $100.6^{\circ}$ and pulse to 104. During the night he slept well and appeared free from pain.

The following morning he did not complain of any pain, but on deep pressure some tenderness was elicited in the right hypochondriac region. The temperature was $100^{\circ}$ and the pulse 98 to 106 . The abdomen was perfeetly soft, and moved uniformly on respiration. On percussion it appeared quite normal. Later in the day his copdition changed, and rather suddenly so. The pulse increased in frequency and reached r3o per minute; the temperature rose to $\mathrm{IO}_{2}{ }^{\circ}$; respirations were unaltered. The abdomen was slightly distended generally; abdominal movements were free, except in the right hypochondriac region, and there the wall was held rigidly fixed and great tenderness was complained of, the remainder of the abdomen being free from all pain and tenderness. On percussion there was an area of tympany reaching from the umbilical level on the right side upwards to the fifth rib in the nipple line, backwards to the mid-axillary and inwards to the mid-lines. There was absence of liver dullness anteriorly. When the patient was turned on to his left side this tympanitic area extended backwards to the scapular line, beyond which level some impaired resonance was obtained over the right base where the entry of air was deficient. Some impaired resonance was also present in the right loin. These signs pointed to a localized collection of fluid gas in the right hypochondrium, and from the situation and the absence of all previous symptoms a perforated duodenal ulcer was suspected.

Operation. - Twenty-eight hours after the initial symptoms a median epigastric incision was made and lengthened eventually to about 5 in On opening the peritoneal cavity some turbid fluid escaped; this was coming entirely from the right side : the remainder of the peritoneum was not infected apparently, and was shut off by sterile gauze packing. The duodenum just beyond the pyloric opening was considerably thickened and adherent to the liver; from amongst these adhesions flutid was seen issuing. The adhesions to the liver were separated and a perforated ulcer exposed in the centre of a very indurated duodenal wall; the perforation was about the size of a threepenny piece. An attempt was made to close the hole with Lembert sutures. The bowel, however, was too friable to permit this. The free edge of the omentum was brought up and stitched around the perforation. The peritoneum was carefully dried as thought. A counter incision was made in the right loin, just below the last rib, and immediately the peritoneal cavity was opened some few ounces of turbid fluid escaped. The anterior wound was closed with the exception of about $x$ in. through which a rubber drainage tube was passed to the surface of the ulcer; the lumbar wound was drained by a rubber tube also. The operation lasted about thirty-five minutes. At the close Oilj of saline solution were put into the median basilic vein. The following morning the patient had a rigor, the temperature reaching 10 $5^{\circ}$ and the respirations 36 . Lung trouble was anticipated, but the alarming symptoms subsided in a few hours and the temperature fell to normal in a few days. The tubes were removed on the eighth day. A few days later a small abscess developed in the abdominal wall, presumably due to stitch infection. This was treated and all went well. Now, seven months later, the man is in perfect health; he has no symptoms of duodenal ulceration, active or healed.

The points of main interest in this case are : (1) The absence of , all symptoms previous to those of perforation. This appears to be the case in about 50 per cent. of perforated duodenal ulcers. It is so common, in fact, that in a middleaged man, in previous good health, who suddenly develops signs of acute perforative peritonitis of the upper abdomen, duodenal ulcer should be strongly suspected. (2) The occurrence of acute symptoms during some severe straining action. This is frequently observed in gastric and not uncommonly recorded in the duodenal perforation also. In the stomach it is easy of explanation. The abdominal wall contracting would tend to compress a full stomach and cause a rupture at any weak spot. Compare the etiology of the cases of spontaneous rupture of the stomach. The condition is somewhat different in the duodenum, and it is difficult to conceive that the strain can cause a perforation in a similar way. In this case, and possibly in others recorded, I think the strain tore adhesions between the duodenum and liver, and thus caused the hole in the duodenum. (3) The length of the "latent period" and the almost entire absence of symptoms in that period. In most acute abdominal conditions after the initial collapse there comes a period in which the patient is comparatively comfortable, but generally some evidence of abdominal disease can be detected. In this case the abdomen was practically free from all evidence of disease ; the pulsewas a little increased in frequency, and the temperature a little elevated, and slight tenderness on deep pressure was present. Signs of peritonitis did not develop until twenty-four hours later. The perforation in the early collapse must have been very small and immediately sealed by adhesions. Later adhesions gave way and allowed of free extravasation. (4) The clinical phenomena pointing to an effusion of gas and fluid in the upper right region of the abdomen. The large area of tympany obliterating the liver dullness, changing its limits on movement of the patient on to the left side, and the general suppleness of the rest of the abdomen produced a gastrointestinal perforation. At the operation no adhesions were found shutting this fluid collection off from the rest of the abdomen, and it is difficult to understand why it did not overflow into the general peritoneal cavity, but it evidently did not. This right renal pouch, the extent of which was first pointed out, I think, by Rutherford Morison, is said to be capable of holding a pint, and in this case it must have had its full complement. (5) The importance of a lumbar drain may be emphasized. I thought I had evacuated the whole collection from the anterior incision, and was surprised to find the escape of more fluid from my lumbar incision. Drainage from the anterior wound alone would have been very imperfect.

\section{CASe II}

Male, aged 37. He was thought to have had an attack of acute appendicitis two years previously, and the history given of this illness strongly suggested the appendix was at fault. Apart from this there was no history of previous gastro-intestinal disease. He was suddenly seized with acute abdominal pain, chiefly on the right side, and the maximum pain was in the right lumbar region. The pain occurred two hours after dinner. Six hours later he was easier, but still had considerable pain in the right side of the abdomen. The abdomen moved freely, with some restriction on the right side; the wall was somewhat rigid here, but not particularly in the iliac fossa. Temperature, 100.20: pulse 80 to 90 . A few houre later the pain increased in severity, the pulse became more frequent, and the temperature was rising. When seen thirty hours after the onset of the symptoms, he was evidently very ill. The temperature was $102.6^{\circ}$, pulse 120 . The abdomen was distended and its movements were very much restricted. The whole of the right half of the abdominal wall was rigid; the maximum tenderness was in the right iliac fossa. There had been no vomiting; the bowels had acted once. Liver dullness was present to the normal extent; some questionable impaired resonance was present in the right loin. He was evidently rapidly getting worse. From the previous attack of appendicitis, the absence of all other previous illness, and, when seen; the maximum pain and tenderness in the right ilias fossa, perforative appendicitis was diagnosed. Pelvic examination was negative.

Operation.-Thirty-one hours after the initial symptoms an incision was made in the right iliac fossa; on opening the peritoneal cavity some turbid, non-smelling fluid escaped; the appendix was immediately exposed and seen to be healthy. A gastric or duodenal perforation seemed therefore most probable, and the abdomen was opened in the epigastrium. A considerable quantity of turbid fluid welled up from the right side. A perforation of the duodenum was discovered about three-quarters of an inch beyond the pylorus. This was closed with a single layer of Lembert sutures. The margins of the perforation were not very healthy and did not hold the stitches well, and consequently the omentum was drawn up and stitched over the site of pertoration. The peritoneum was then cleansed. The peritonitis appeared to be quite localized to the right side of the abdomen. A counter-opening was made in the right loin and a tube was passed through this. The opigastric and iliac incisions were drained with gauze. The operation lasted forty minutes. The patient made an uninterrupted recovery. The gauze drains were dispensed with on the seventh day and the tube on the tenth day. Four months later he is in very fair health, but suffers from what he terms "indigestion"-that is, occasional pain some two to three hours after food, which lasts a very variable time. 
This is unaffected by diet. Presumably this is due to the unhealed duodenal ulcer.

The interesting feature about this case is the similarity to

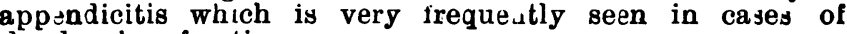
du denal perforation.

$$
\text { CaSE III }
$$

Male, aged 53. He suffered from "indigestion" for three years. This "indigestion" was pain about half an hour after meals; it was not constant; he was often free for weeks together. The pain lasted about two hours, and was often relieved by taking food. There was no vomiting; he suffered much from flatulence. For three weeks the pain had been getting worse, and was particularly so at night-time; it then had no relation to food. He was suddenly, whilst walking along the street, seized with acute abdominal pain and collapse; he was able to w ilk a few yards to a doctor's house, where he lay very collapsed for about an hour, being quite unable to move. He was then carried to the hospital, merely a few yards off. Oa admission he was evidently in considerable pain. Temperature $96^{\circ}$, pulse 90 , respirations 26 . The abdomen was flattened, moved only slightly on respiration; it was generally rigid and markedly tender in the right hypochondriac region. Then I saw him, about eight hours later, he was still in considerable pain; the abdomen was moving more freely, but there was still some tenderness in the right hypochondriac region. Temperature had risen to $99^{\circ}$, pulse 90 . No abnormal percussion changes were observed. There had been no vomiting. He was the subject of chronic bronchitis and emphysema, and had been under treatment for many months for bronchial asthma. From the history and clinical sigas I suspected a perforated duodenal ulcer, and advised operation. This was absolutely refused. A few hours later he consented to the operation, and I again saw him. At this time every abdominal symptom had disappeared; his pulse was $9 \circ$, and temperature $99^{\circ}$. I doubted $\mathrm{my}$ original diagnosis, especially when he informed me that he had frequently been like it in the past few weeks. No food was allowed by the stomach. The pain in the right hypochondrium returned, however, and with it the rigidity; the pulse rose slightly to 100 , and the temperature $99.8^{\circ}$. These facts, together with the severe initial collapse, made me determine to operate.

ONeration.-Twenty-two hours after the initial symptoms a median epigastric incision was made about 5 in. long. On opening the peritoneum 3 or $4 \mathrm{oz}$. of turbid lymph escaped from the right hypochondriac region, the lower stomach and intestines in this region were coated with lymph. In the flrst part of the duodenum was a tiny perforation surrounded by very indurated bowel wall. The surrounding bowel would not hold sutures and therefore an omental graft was sewn over the perforation with a few interrupted sutures. The peritonitis was quite localized; the peritoneum was carefully cleansed; a lumbar incision was made and the peritoneum drained from hore by a rubber tubs; the site of perforation was covered with gauze, the epigastric incision was closed with the exception of the gauze drain. The operation lasted thirty-five minutes. The following day be developed acute bronchitis which gave rise to considerable anxiety for a few days. It gradually subsided, horvever. The skin sutures gave way and caused considerable gaping. The lumbar wound healed in a fortnight; the epigastric sinus discharged for some fwo weeks longer, and the wound was very slow in healing. All his "indigestion" left him for some three or four weeks, but then he suffered again from discomfort and flatulence.

The symptoms of gastric ulcer are often extremely uncertain, and it is not very infrequent for the first symptom of an ulcer of the stomach to be the initial shock of acute perforative peritonitis. It is quite a common experience to be told that the only symptoms previous to the perforation of a gastric ulcer were occasional vague pains of "indigestion." But yet the majority of cases of gastric ulcer which perforate have previously suffered from symptoms which should lead one very strongly to suspect the existence of the ulcer. This is not the case in perforated duodenal ulcer.

To Collin, Perry and Shaw, Schwartz, Weir, and others, we are indebted for papers on the subject of duodenal ulcer in one or more of its phases. From a series of cases recorded in more recent times, some of which are included in the statistics of the above-mentioned authors, some of which have only been published since these papers, and a few are previously unrecorded, I deduce the following facts :

Sex : Males 47 ; females 3 .

Between 18 and 20 years of age perforation occurred 6 times.

$$
\begin{aligned}
& 20 \text { and } 30 \quad \text { " " }, \quad 14 \text { times. } \\
& 30 \text { and } 40
\end{aligned}
$$$$
40 \text { and } 50
$$

These figures apply only to the age at which perforation occurred; it is impossible to say at what age the ulcer developed, since so many are free from symptoms.

In every case in which mention is made of the fact the ulcer appears to have been chronic.

In 26 cases previous symptoms are stated to have been entirely absent, the patients being in very good health previous to the occurrence of perforation. Of the 24 patients who suffered from previous symptoms, in 16 the only complaint was occasional or continuous "indigestion " of some duration. This means they suffered from pain after food occasion- ally, or more or less regularly, but the pain had no constant time-relation to the in sestion of food. Sometimes pain was experienced immediately, and sometimes not for three or four hours after a meal. Pain was sometimes replaced by discomfort merely, and a sense of distension of the upper abdomen. Pain is sometimes described as being worse on the right side of the epigastrium, but this is so inconstant, even in the same patient, that it is of little value in diagnosis. In 4 cases the pain was very severe, occurring in paroxysms. These paroxysms occurred at such irregular intervals after meals that it seems very much open to doubt whether they had any dependence on food. A more reasonable explanation seems to be a stretching of adhesions. Whether this were so in these cases is not mentioned, but the paroxysms resemble very much the pain we associate with gastric adhesions. Tenderness in the right hypochondrixc region is mentioned only three times. Other features concerning the pain have been noticed by various observers, but were not present in this series of 50 cases. Thus Boas describes pain radiating to the back: Marocco describes pain referred to the tenth and twelfth dorsal vertebrae; the latter observer and Schwartz have noticed pain referred to the right shoulder. Poynton records the case of a patient where pain was so severe as to resemble the erosion of vertebrae from an aortic aneurysm. Many observers lay great stress on the time of the occurrence of pain after the ingestion of food. Many of the latter observations, however, are from patients in whom an ulcer has never been proved to exist, either by operation or post-mortem examination, and in a disease like duodenal ulcer, where the symptoms are so indefinite, it is quite useless to attempt to build up statistics in any other way. In this series of cases which I have collected pain certainly never had this constant relation to food which is said to be so characteristic by many authorities. In fact, the time of occurrence of pain after meals, the situation of the pain more to the right than the left side of the middle line, the areas of referred pain, and the tenderness on the right side are all of very little value in the diagnosis of a duodenal ulcer.

In 8 cases only haemorrhage occurred; in 4 haemorrhage was the only symptom; in 4 haemorrhage and some occasional epigastric discomfort both existed; in 3 cases the blood was vomited alone; in 2 cases it was passed by the bowel alone; and in 3 cases haematemesis and melaena both occurred. Vomiting apart from haematemesis was only recorded four times.

This apparent absence of all symptoms previous to perforation in about half the cases is borne out by other observers. Thus Schwartz found that 20 out of 25 patients who perforated from a duodenal ulcer were in apparent good health, or so slightly indisposed that they had never consulted a physician previous $t$ ) the occurrence of perforation. Weir found that in 25 out of 51 the first indication of the ulcer was perforation.

The mortality of duodenal ulceration may be roughly estimated from the following figures:-Chvostek, in 63 cases of duodenal ulcer, noticed perforation 27 times; in Collin's 262 cases it was present in 18r; Oppenheimer describes it in 38 out of 79 cases. It can be gathered from these statistics what a very frequent complication perforation is. It is impossible to say in what exact percentage it occurs, but from these figures and from the fact that the majority of cases run a chronic course, and that only comparatively rarely has cicatrization been noticed (39 out of $262-$ Collin), the percentage must be a very high one; in fact, it so frequently occurs, that it may almost be said to be the one constant sign. Perforation probably occurs in about half the cases of duodenal ulcer.

Rejults of Surgical Treatmant.

The results of the surgical treatment of perforated duodenal ulcer, although encouraging, leave a lot to be desired. The recovery after operations taken from a large number of statistics from all sources, and including all varieties of perforation and peritonitis, is something like 20 or 25 per cent. About 65 per cent. of those operated upon in the first twelve hours recover; about 12 per cent. of those operated upon between the twelfth and twenty-fourth hour recover, but after this only a very small proportion. These results are improving every year, perhaps owing to the earlier recognition of perforative peritonitis, and also to the improved technique marformed for the same. Yet the improvement is slight, and d codenal ulcer still has a very high mortality. 
Summarizing the above facts, it may be said that the symptoms of duodenal ulcer are very indefinite, and often consist of no more than.vague "indigestion" pains; that in about 50 per cent. of cases perforation is recorded as the initial sign; that taking all varieties of perforation the mortality is about 75 per cent. - this is considerably lessened the earlier the case comes under surgical treatment; that this mortality is being slightly lessened year by sear, but only by the earlier recognition of perforative peritonitis and improved operative technique; that the diagnosis of duodenal ulcer is as yet very uncertain; that the comparatively high mortality from duodenal ulcer can only be lessened by surgical treatment before perforation, or by an even yet more certain diagnosis of acute perforative peritonitis.

\section{Clinical Course.}

Duodenal ulcers run a chronic course; cicatrization is very infrequently noticed. Perforation occurs in about 50 per cent. of cases, and this still has a very high mortality. The results of operation for duodenal ulcer previous to perforation are, as recorded cases show, most brilliant. Pain disappears, haemorrhage ceases, health is rapidly improved. The mortality is very low, and I can find no recorded case where perforation has occurred after gastro-enterostomy. With these facts before us, therefore, the treatment of a patient whom we strongly suspect to be suffering from duodenal ulcer becomes entireiy surgical; and even more, with a reasonable suspicion of duodenal ulcer, the treatment should be surgical.

A duodenal ulcer may perforate so as to permit of free extravasation of intestinal contents into the periteneal cavity. This is generally seen in those cases which perforate shortly after a meal. If only a little fluid escape the symptoms are often very much modified, and are those of local peritonitis but the general peritoneal cavity is not shut off. Perforation may occur, adhesions form, and a periduodenal abscess arises. In the first class of case the symptoms are those of any acute perforative peritonitis of epigastric origin, and the diagnosis depends on previous history. Pain is not more marked on the right than the left side, or with such inconstancy as to be valueless in the diagnosis. A man of middle age with absence of previous symptoms would suggest a perforated duodenal ulcer. The second class of case is the more frequent. The early symptoms here are the same as in the former class, but generally less intense. There is, however, in every case an acute onset-sudden severe pain and some collapse; vomiting is infrequent. It is in this class of case that the pain from the very earliest is so often experienced in the right upper region of the abdomen.

But sometimes here, also, the pain at the onset is general or most intense around the umbilicus, but after a short time it becomes referred almost entirely to the right hypochondriac region. The early pain is not in these cases in the lower abdomen. The prognosis in perforated duodenal ulcer depends primarily on the extent of peritonitis, which bears some relation, but an inconstant one, to the duration of the perforation, and also to the amount of fluid in the stomach at the time of perforation. In Cases I and II the patients were being fed by the mouth for some hours after the perforation occurred, and at the operation there was an extensive local peritonitis with much extravasation, whereas in Case III nothing was given by the mouth, and there was a very little extravasation. The successful result of the operation in each case is due to the fact that the peritonitis was localized, although operation was performed twenty-eight, thirty-one, and twenty-two hours after the perioration.

Operation must be performed in quite the early stages, certainly before the peritonitis is general, and also before there is much local extravasation. A person in previous good health who is suddenly seized with acute abdominal pain and severe collapse, is in the majority of cases suffering from some grave disease in the abdomen or chest. The chest may be excluded in a few hours if we pay attention to the following points: The absence of true abdominal rigidity, the temperature, and the pulse-respiration ratio, and other evidences of acute pulmonary trouble-for example, the aspect of the patient and cough. The severe tearing character of the pain, the severe collapse, true abdominal rigidity, the facial aspect, the increased pulse-frequency in the early stages, all point to a peritoneal lesion. The pain abates somewhat, the pulse often diminishes, rigidity however persists, and the temperature rises. In this period, which is often referred to as the "latent period," it is most rare for all symptoms to disappear unless the patient be under the influence of any drug.
Sometimes, however, they are so mild as not to be complained of by the patient.

A few years ago I remember a young man was thrown from. his bicyle and fell on the handle-bars, the handle-bar striking him in the abdomen. He was severely collapsed for about half an hour, and then resumed his journey, a distance of two miles. some few hours later he had evident peritonitis, and operation showed that an extensive tear in the small intestine with wide extravasation of intestinal contents. Every one has seen patients walk into the hospital during their latent period, not in any marked evident distress. During this latent period some signs of peritonitis are generally evident, and they are true abdominal rigidity, abdominal pain and tenderness, and some fever. The pulse frequently has fallen after the initial shork, and it is not a true guide. In these cases of subacute perioration some rise in temperature after the initial collapse is very constant. It may be only $100^{\circ}$ or possibly less, but is very significant. Immediately there is evidence of any peritonitis the abdomen must be opened. Delay on the off-chance that the peritonitis will result in a localized abscess is not justifiable. We can never foretell this in any case of peritonitis, and localized abscesses from duodenal perforation are notoriously fatal. A most careful watch must be kept on all patients suddenly afflicted with acute abdominal pain and collapse. Food by mouth must be withheld, and morphine must not be given.

\section{Prognosis.}

The prognosis of perforated duodenal ulcer at the present day is not so good as perforated gastric ulcer. This is merely because in perforated gastric ulcer the early symptoms are more severe, and signs of peritonitis appear earlier. A duodenal usually perforates subacutely, the early symptoms are less intense, and signs of peritonitis are longer delayed ; they are milder, and we do not appreciate, until unfort unately too late, their existence. Too much stress cannot be laid on the importance of the initial collapse which is present. in some degree in all perforations of the gastro-intestinal tract. The very fact that a duodenal ulcer perforates subacutely and the peritonitis will often remain. localized and the extravasation is so limited, should make. the prognosis of perforated duodenal ulcer comparatively good. But this, unfortunately, is not so. A clear appreciation of the early signs is imperative. We must recognizethat sudden acute abdominal pain and collapse means something - and something, generally, of a very serious natureand no pains must be spared to elucidate their origin, and once we have slightest suspicion of peritonitis the abdomen must be opened. In Case III I was almost entirely guided by the early signs in the diagnosis of perforative peritonitis ; for, when I saw him first, there was merely a suspicion of peritonitis. My regret is that I did not operate earlier, but, as events showed in this case, it made no difference, but it might have done so. I am well aware that more than one abdomen has been opened on the suspicion of perforative peritonitis, and nothing has been found. But what a very small minority these are-so small as to be absolutely of no value.

Many diseases may, of course, be confounded with a perforated duodenal ulcer. The chief among these are perforated gastric ulcer, acute peritonitis of gall-bladder origin, suppuration in connexion with the colon, and acute appendicitis. Peritonitis from the gall bladder and colon can generally be differentiated by the previous history and the onset of the symptoms. Acute appendicitis is frequently confused with duodenal perforation, as in Case II. This is easily understood from anatomical considerations, the extravasation gravitating down along the colon, and collecting in the right iliac fossa. So frequent is this, that in 17 out of 50 cases acute appendicitis. was diagnosed, and in ro additional cases it was suspected: Peritonitis should be diagnosed before this widespread extravasation has occurred.

A perforated duodenal ulcer has only on rare occasions been diagnosed.

I. Symptoms of perforative peritonitis occurring in a person in previous good health, particularly a male subject between 30 and 50 years of age, the early symptoms being marked in the upper region of the abdomen, should excite a suspicion of duodenal perforation.

2. Should these symptoms occur in a person who has previously complained of gastric disturbance, however vague it may be, the suspicion is greater. In the very acute perforating duodenal ulcers the differential diagnosis from gastric perfora- 
tion will be impossible. Gastric ulcers generally perforate in young anaemic women; duodenal ulcers in men between $3^{\circ}$ and 50 . But this is by no means constant.

3. Symptoms of acute perforative peritonitis of epigastric origin and spreading down to the right iliac fossa, simulating an acute appendicitis, is frequently seen in duodenal perforation, but very infrequently in gastric perforation. In these cases it can generally be noticed that the early signs are referred to the upper abdomen, the iliac fossa is only secondarily involved. Rigidity at first is in the hypochondriac region, and only later in the iliac region. The initial shock is more intense generally in acute perforative appendicitis, and septic poisoning is seen earlier in the history of the case.

4. An acute appendicitis may travel from the iliac fosea to the right hypochondriac region, and thus simulate a duodenal perforation. The earliest signs here are in the right iliac region, and only secondarily located in the hypochondrium. The similarity of duodenal perforation to acute appendicitis must be borne in mind, and by attention to the onset, the early site of pain, the area of rigidity and the initial higher fever in appendicitis, a correct diagnosis will generally be arrived at.

5. The occurrence or not of haematemesis or melaena should be inquired into. A previous history of melaena only suggests a duodenal perforation.

6. It is easier to exclude other causes of perforative peritonitis than a duodenal ulcer, and by exclusion a perforated duodenal ulcer ought to be more frequently diagnosed.

\section{AFTER-Historx.}

An interesting point in connexion with perforated duodenal ulcer is the aiter-history of the patients who recover from operation. Several interesting questions arise. Should symptoms have been present before the operation, do they continue? Should symptoms have been previously absent, do they arise later on, after the successful closire or the perforation? Does perforation ever occur again, and are these patients still in great danger from this catastrophe? Does the treatment stop with the suture of the ulcer, and ought not further surgical means be taken to secure healing of the ulcer?

The after-records of these patients is almost entirely wanting in surgical literature. Occasionally a note after some months is added that some "indigestion still remains," or something to that effect, but there are, as far as I know, no recorded after-histories from which these questions might be answered. I cannot find the records of a case which has perforated a second time. Viewing the question generally from the statistics I have given above it would seem that our treatment should not stop at the successful treatment of the perforation. Duodenal ulcers are very chronic. They show a very little tendency to heal naturally : merely sewing up a hole in the floor of an ulcer, or placing a piece of omentum over it cannot increase this slight tendency towards natural healing. Theoretically the dietetic treatment of the patient after the operation might do so, but we have no evidence to show that it does. The dietetic treatment of a chronic gastric ulcer is very unsatisfactory, and we should expect the same in duodenal uicer. If we compare chronic gastric ulcers which perforate and are successfully operated upon, and follow these cases alterwards, in many - I should estimate the majoritysymptoms of an unhealed ulcer persist. Looking at the question from the statistics of duodenal ulcer, and by comparison with gastric ulcer, in all probability the ulcer remains opened. What is ( 1 ) the inconvenience to the patient of this unhealed alcer, and (2) what is the danger to life? The inconvenience to the patient can only be measured by his symptoms. Should efmptoms versist or arise further surgical messures should be undertaken, other things being equal. The danger to life is perforation. Although $I$ can find no evidence of this having occurred twice in the same patient, I see absolutely no reason why it should not. Realizing the serious condition of a patient with a duodenal ulcer, I advocate further interference ito secure the closure of the ulcer. With this end in view, after explaining to two of my cases the position, I advised a further operation. Both patients, however, preferred to run the risk, and declined. The third case was so extremely ill from acute bronchitis after the operation that it was not suggested to him.

$A$ word may be said here with regard to the closure of the perforation. In the majority of these cases there is considerable induration around the perforation, and to close by sutures means puckering the wall and consequent narrowing of the lumen of the bowel. A better method, I think, will be found in an omental graft, if this can be secured, and, failing this, a coil of small intestine. Omentum is perfectly safe; it is easily secured by a few stitches. In fact, probably laying the omentum over the site of perforation and keeping it in position with gauze packing would be sufficient, if time were a great object or for any reason suturing were rendered very difficult. An omental graft would, theoretically, be a more protective barrier against a future perforation than suture.

A duodenal ulcer may perforate, adhesions form, and local suppuration occur. These localized abscesses from duodenal perioration are very rare. Bainbridge in 1903 collected all cases which had been recorded up to then, and found only 26. I have seen one case since. Of the 27 cases, 23 died without a diagnosis being made, in 2 a diagnosis was made at the operation, in 2 a correct diagnosis was made; one of these was operated upon, the after-history is not given, and in the second the condition of the patient prevented any interference. The age of the patients varied from 15 to 56 years, but the majority occurred at the age at which periorated duodenal ulcer is most frequently seen. Analysing the few cases where the previous history is recorded, about 50 per cent. had suffered from gastric trouble, which is mentioned as digestive disturbance, digestive disorders, dyspepsia, or stomach trouble. In one case blood had been passed per rectum a year before, a second case occasionally vomited blood; 6 cases have been operated upon : in 3 cases the abscess was merely opened; and in 3 cases the perforation sutured. Moynihan records a successful case in which he diagnosed the perforation during the operation, closed it, and drained the abscess. Lowson records a case where he diagnosed the condition before operation, sutured the perforation, and drained the abscess. The after-history of the case is not given. A fatal result has attended all the other cases.

The diseases most liable to be confounded with this condition are appendicitis, acute inflammation or perforation of the gall bladder, and suppuration in connexion with the colon. More than once the trouble was thought to have been entirely above the diaphragm. The points in the diagnosis upon which I lay stress are the following:

I. The absence of previous symptoms.

2. If there be a history of previous trouble, its exact nature should be elicited-whether gastric or suggestive of gall stones or appendicitis, or of obstruction from carcinoma of the colon.

3. The exact situation of the early pain. This is usually relerred to the right epigastric or hypochondriac region, higher than that of appendicitis.

4. The early rigidity and tenderness over the upper half of the right side of the abdomen.

5. The early tenderness on deep pressure over the lower ribs posteriorly and laterally.

6. The marked increase of pain on deep inspiration in quite the early stages.

7. The frequently observed clinical signs of elevation of the right half of the diaphragm.

8. These abscesses very frequently contain gas, thus differing from those of the gall bladder. Gaseous abscesses originating from the gall bladder are most rare, and in carcinoma of the colon and in appendicitis a detectable quantity of gas in the early stages is most unusual.

\section{THE OPERATION OF GASTRO-DUODENOSTOMY ; ESPECIALLY IN REFERENCE TO FINNEY'S OPERATION OF GASTRO-PYLO- DUODENOSTOMY.}

Bx DONALD ARMOUR, M.B., F.R.C.S.,

Arris and Gale Lecturer, Royal College of Surgeons; Assistant Surgeon, West London Hospital ; Burgeon, Belgrave Hospital for Children.

Trat the operation of gastro-jejunostomy is the one practically always employed in this country when it is desired to make an anastomosis between the stomach and small intestine for whatever cause, is clear from a search through the literature published upon the subject. In none of the textbooks, save one, is the operation of gastro-duodenostomy mentioned except in connexion with that of pylorectomy ; and one goes so far as to say, "gastro-duodenostomy being only employed after a pylorectomy has been performed." That such statements need revision is obvious in the light of recent work published from various clinics abroad. 